\title{
Vorwort zu „Sichere Lebensmittel: von der Früherkennung bis zur Sanktion“
}

\author{
Andreas Zapf ${ }^{1}$
}

Published online: 11 January 2017

(C) Bundesamt für Verbraucherschutz und Lebensmittelsicherheit (BVL) 2017

In den letzten Jahren ist das Thema Lebensmittelsicherheit zunehmend in den Fokus der Öffentlichkeit gerückt. Fortschreitende Globalisierung, komplexe rechtliche Vorgaben, neue Technologien, Änderung von Verbrauchererwartung, Verzehrsgewohnheiten und Lebensweise sowie ein gesteigertes Medieninteresse stellen Behörden, Wissenschaft und Wirtschaftsbeteiligte vor immer anspruchsvollere Aufgaben. Unter dem Motto ,Sichere Lebensmittel: von der Früherkennung bis zur Sanktion“ fand am 18. und 19. Oktober 2016 der 2. LGL Kongress Lebensmittelsicherheit des Bayerischen Landesamtes für Gesundheit und Lebensmittelsicherheit (LGL) in Erlangen statt. Der Kongress bot praxisnahe Informationen zu aktuellen Themen der Lebensmittelsicherheit und Lebensmittelüberwachung. Dabei wurde ein Bogen von lebensmittelrechtlichen, analytischen und verfahrenstechnologischen Fragen bis hin zu künftigen Ernährungsformen gespannt. Der erste Tag wurde durch drei Plenarvorträge hochkarätiger Experten der EFSA, des BfR und des BVL eingeleitet und stand unter dem Schwerpunkt „Risikobewertung und Risikokommunikation“. Die unterschiedlichen Aspekte des Lebensmittelbetrugs, ein zentrales Thema, nicht nur der amtlichen Lebensmittelüberwachung, wurden umfangreich beleuchtet.

Dr. Andreas Zapf

Praesidentenbuero@lgl.bayern.de

1 Bayerisches Landesamt für Gesundheit und Lebensmittelsicherheit, Eggenreuther Weg 43, 91058 Erlangen, Germany
Am zweiten Tag fanden zwei spannende Diskussionsforen statt, die sich mit den Themen „Information der Öffentlichkeit“ und „Grenzen der Lebensmittelsicherheit“ beschäftigten. Dafür konnten kompetente Stakeholder aus Industrie, Verbraucherschutz und Politikgewonnen werden. Komplettiert wurde das Programm durch insgesamt 16 Vortragsblöcke, die den breiten Zuständigkeitsbereich des LGL abdeckten. Beispielhaft seien genannt: „Alternative Ernährungsformen, Pestizidanalytik, Non Food, Zoonosen, Neue Analysemethoden, Lebensmittelrecht, Qualitätssicherung, Toxikologische Aspekte und Authentizität“. Die in den Vorträgen angesprochenen Themen wurden durch 20 Posterbeiträge ergänzt.

Das wissenschaftliche Programm zeigt deutlich die Vernetzung des LGL als zentrale Fachbehörde des Freistaates Bayern, die einerseits nationalen und internationalen Netzwerken angehört, andererseits als Fachbehörde und zentrale Ausbildungs- und Forschungseinrichtung im intensiven Dialog mit den Landkreisen und Regierungen steht.

Begleitend zum Kongress halten Sie nun den vorliegenden Supplementband in den Händen. Ich hoffe, dass die ausgewählten Beiträge zur Vertiefung der Themenschwerpunkte beitragen werden. Ich bedanke mich bei allen Referentinnen und Referenten, die unserer Einladung gefolgt sind und einen Artikel verfasst haben.

Ich wünsche uns allen eine spannende Lektüre!

Ihr Andreas Zapf 